\title{
Extension of the ILDM method to the domain of slow chemistry
}

\author{
V. Bykov *, U. Maas \\ Institut für Technische Thermodynamik, Karlsruhe University, Kaiserstraße 12, D-76128 Karlsruhe, Germany
}

\begin{abstract}
This work focuses on the construction of reduced kinetic models and the use of these models for the simulation of combustion processes governed by strongly coupled thermo-chemical and convection/diffusion sub-processes. The ILDM method is used to reduce the system dynamics in the composition space to lower dimensional manifolds. This manifold approximates an invariant system manifold of slow motions. A modification of the ILDM approach based on a special system representation is suggested, which allows to use an ILDM of low dimension even in cases where the standard formulation would require a high dimension. In this way difficulties of generating relatively high dimensional ILDMs are overcome. The approach allows a more accurate description of coupled thermo-chemical and transport sub-processes. When the processes are split a method of extension of the ILDM manifold to cover all the domain of interest in the full state space is suggested. It is based on the assumption of slow chemistry inside the low-temperature zone of the flame. To verify the approach 1D stationary free flat laminar flames are investigated. It is shown that the approximation allows a representation of the full system dynamics governed by detailed chemical kinetics and molecular transport.

(c) 2006 The Combustion Institute. Published by Elsevier Inc. All rights reserved.
\end{abstract}

Keywords: ILDM; Reduction; Invariant manifolds

\section{Introduction}

During the last two decades there has been an enormous progress in the numerical modeling of laminar and turbulent flames (see e.g., [13] for references). However, despite the steadily increasing computational power and increasing efficiency of numerical methods detailed calculations of technical combustion processes are in most cases computationally prohibitive, especially when combustion processes are considered with detailed

\footnotetext{
* Corresponding author. Fax: +497216083931.

E-mail address: bykov@itt.uni-karlsruhe.de (V. Bykov).
}

chemical kinetics models, where the timescales may differ by orders of magnitude ( $1 \mathrm{~s}$ to $10^{-10} \mathrm{~s}$ ) and the number of species and reaction steps exceeds 100 and 1000 , respectively. The disparity in timescales leads to a stiffness of the mathematical models that makes their numerical treatment problematic, and large computational resources are needed for an accurate integration of such systems. Hence, in order to overcome these difficulties methods for reduction of both dimensionality and stiffness of the systems describing combustion problems are frequently developed and used (see e.g., [4])

At present there are a number of asymptotical/ analytical and numerical tools able to treat the multi-scale system of differential equations. 
An incomplete list includes the computational singular perturbation (CSP) method [1-3], the method of integral or invariant manifolds (MIM) [5-8] and the functional iteration method [9]. These methods have been constantly improved, and many variations are now available. One other technique that has been developed to simplify chemical kinetics is the ILDM (intrinsic low dimensional manifolds) method [10]. The method allows an automatic exploration of the hierarchical structure of the system by a spectral decomposition of the Jacobian of the source term and an approximation of an invariant system manifold. The method has a mathematical background, and it was shown in a number of works [15-18] that the ILDM provides a good approximation of the invariant manifold of slow motions (up to the second order [16]), when a gap condition of the Jacobian's eigenvalues is satisfied. This condition will be discussed below and it means that the eigenvalues are separated into two subgroups according to their orders of magnitude.

In spite of many advantages of ILDM type automatic reduction models there are principal, fundamental drawbacks of such approaches. The first is connected with the fact that an attractive property of the manifold containing the reduced system dynamics does not exist everywhere in the whole domain of interest in the state space. It means that the system dynamics cannot be reduced by the ILDM in some part or in some sub-domain of the whole domain. A lack of a rigorous methodology for the separation of slow and fast chemistry domain is the second major drawbacks. For that reason, by now, there are a number of "tricks" which make the implementation of the ILDM technique possible. Examples are fake state space relations in the domain where the ILDM does not exist, or flamelet prolongation of the ILDM [21]. In the following we suggest an algorithm which overcomes these drawbacks in general case. Moreover, it allows systematically treating the coupling of the chemical and transporting sub-processes by an adaptive increase of the dimension of the manifold. In the following we shall outline the concept and verify it using the simple syngas/air flame, which has been the subject of previous studies. This is a quite simple test case, but nevertheless includes all the specific problems of the dynamics of combustion systems. An application for the chemistry of combustion of high hydrocarbons (which have been studied sufficiently in the context of the ILDM approach, see $[10-14])$ is straightforward.

\section{Mathematical description}

A key inspiration of the work is the concept of a domain decomposition that is introduced to treat the transport and chemical terms separately.
This together with a modification of the ILDM that allows to calculate ILDMs of high dimensions very efficiently improves the performance of the ILDM significantly. The suggested modification of the ILDM is based on a general framework that has been developed in a number of works (see for instance $[17,18]$ ), where the question of system reduction based on a decomposition into fast/slow subsystems technique is intensively studied.

\subsection{A brief description of ILDM and suggested modifications}

To present mathematical and geometrical essence of the suggested technique let us define a vector $\psi=\left(\psi_{1}, \ldots, \psi_{n}\right)$ characterizing the thermo-chemical state of the system, here $\psi_{i}$ represents such thermodynamic quantities as the pressure of mixture, the enthalpy, the mass fraction of chemical species, etc. In these vector notations a system of governing equations for reacting flow can be symbolically written as

$$
\frac{\partial \psi}{\partial t}=\mathrm{F}(\psi)+\mathrm{G}\left(\psi, \nabla \psi, \nabla^{2} \psi\right), \psi \in \Omega \subset R^{n},
$$

where the first term related to chemical kinetics (source term) and the second one describes transport processes (convection/diffusion term).

If the spectral gap condition Eq. (2) is satisfied, that is eigenvalues $-\lambda_{j}, j=1, \ldots, n$ of the source term Jacobi matrix $-F_{\psi}$ are separated into two subgroups

$$
\begin{aligned}
& i=1, \ldots, n_{\mathrm{s}} \quad k=n_{\mathrm{s}}+1, \ldots, n_{\mathrm{s}}+n_{\mathrm{f}} \quad n_{\mathrm{s}}+n_{\mathrm{f}}=n \\
& \left|\operatorname{Re}\left(\lambda_{i}\left(F_{\psi}\right)\right)\right| \leqslant a \ll b \leqslant\left|\operatorname{Re}\left(\lambda_{k}\left(F_{\psi}\right)\right)\right| \quad \operatorname{Re}\left(\lambda_{k}\right)<0,
\end{aligned}
$$

where the ratio $-\varepsilon=a / b$ reflects the difference in time scales, then the locally invariant subspaces related to these sub-group eigenspaces define corresponding projection operators $-Z_{\mathrm{f}}, Z_{\mathrm{s}}$ :

$$
F_{\psi}=\left(Z_{\mathrm{s}} Z_{\mathrm{f}}\right)\left(\begin{array}{cc}
\Lambda_{\mathrm{s}} & R \\
0 & \Lambda_{\mathrm{f}}
\end{array}\right)\left(\begin{array}{c}
\tilde{Z}_{\mathrm{s}} \\
\tilde{Z}_{\mathrm{f}}
\end{array}\right), \quad\left(Z_{\mathrm{s}} Z_{\mathrm{f}}\right)\left(\begin{array}{c}
\tilde{Z}_{\mathrm{s}} \\
\tilde{Z}_{\mathrm{f}}
\end{array}\right)=I_{n \times n} .
$$

These projectors define both the system decomposition and reduced system dynamics. According to the ILDM method a manifold containing the slow system dynamics is given as the manifold that annihilates the sub-processes spanned into the fast subspace:

$\tilde{Z}_{\mathrm{f}}(\psi) F(\psi)=0$.

To solve Eq. (4), determining the ILDM manifold, is not a simple task especially for highly dimensional manifolds due to the matrix's $\tilde{Z}_{\mathrm{f}}$ definition. Furthermore during the calculation of the ILDM the eigenvalue decomposition is 
computationally demanding. Therefore, to reduce the level of nonlinearity and simplify the equation the fact that the ILDM is close to the first order approximation defined by standard perturbation theory $[17,18]$ is used. The major output of the analysis performed in [18] is the following. The ILDM manifold produces a reasonable approximation whenever there is an almost constant matrix field transforms the original system to the SPS form. Let us describe this fact briefly and present the suggested modifications. If the theoretical framework of the SPS [5-8] is applied then the simplified version might be given as

$\tilde{Z}_{\mathrm{f}}\left(\psi_{0}\right) F(\psi)=0$.

This corresponds to some zero order approximation of the invariant system manifold when the transport term in Eq. (1) is neglected and the system small parameter tends to zero. Here the eigenspaces are defined at some reference point $\psi_{0}$ that must be additionally specified. Consequently the main source of complexity and nonlinearity is sufficiently concentrated in $F(\psi)$ and the eigenvalue problem has not to be solved on each attempt to find the point on the manifold Eq. (5). To improve performance of the modified version approximations of an invariant manifold of high order of magnitude can be of use, for instance, the implicit form of the first order approximation becomes

$\tilde{Z}_{\mathrm{f}}\left(\psi_{0}\right) F_{\psi}(\psi) F(\psi)=0$.

Additionally, the reference point produces the decomposition might be changed according to the local structure of decomposition. In another words, fixed decomposition may be checked and corrected by choosing another reference point$\psi_{0}$ during the generation procedure of the ILDM table.

\subsection{Domain decomposition and general methodology}

As a motivation and illustration of the domain decomposition approach let us look at the projection of stationary solutions of flat syngas/air flames onto the $\mathrm{CO}_{2}-\mathrm{HO}_{2}$ plane shown in Fig. 1. This system has been the subject of previous studies and the reader is referred to [12] for detailed information. In Fig. 1 different system trajectories are plotted which correspond to different compositions of the unburnt mixture, which nevertheless have the same specific enthalpy and the same element composition, and therefore the same equilibrium value. The figure shows the typical behavior of stationary solutions of combustion problem. They all start initially at different mixture composition points and each of them follows the so called "mixing" line until at a boundary it enters the reaction zone in the flame front,

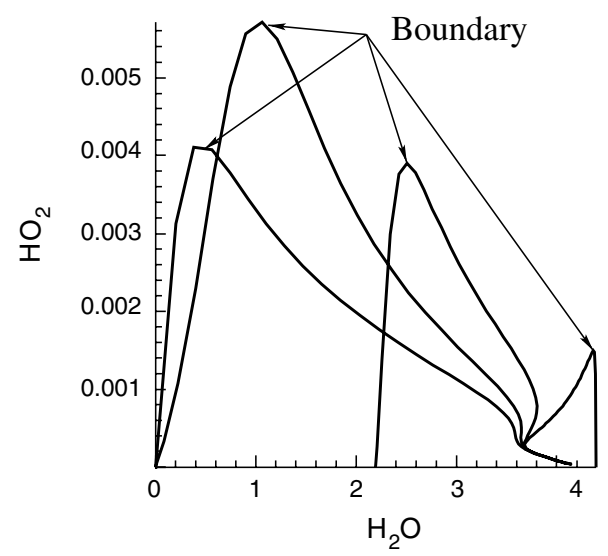

Fig. 1. Projections of the state space onto $\mathrm{H}_{2} \mathrm{O}-\mathrm{HO}_{2}$ planes. Solid lines are stationary solutions.

which can be seen from the rapid change of the direction the trajectories.

To use this behavior, let us assume that the domain of interest can be separated into three different sub-domains such that in the first one $\Omega_{1}$ (see sketch on Fig. 2a) the chemical kinetics governs the system dynamics, which means that the fast chemical processes always equilibrate towards some low dimensional manifold which contains the slow system dynamics and interaction of the slow sub-processes with convection diffusion ones. In the second domain $\Omega_{2}$ the chemical and convection/diffusion processes are strongly coupled or in other words the terms in Eq. (1) have the same order of magnitude. The third one $\Omega_{3}$ is the domain of very (infinitely) slow chemistry where the chemical source term is negligible and the system dynamics is governed by convection/diffusion only.

Typically, the system trajectory in the state space links the equilibrium point, which always belongs to the ILDM manifold and therefore to $\Omega_{1}$, with the initial point (the unburnt point which corresponds to a given boundary condition) and is usually located in $\Omega_{3}$ (chemistry is "slow"-source term is exponentially small (see Fig. 2)).

Note that there might be of course fast chemical processes which are fast everywhere in the domain. For the analysis we can, however, assume that they are already split off before the analysis (e.g., by the standard ILDM concept), and the system dimension is simply reduced beforehand.

Accordingly, three different situations depending on the location of the initial point must be accounted for. Fortunately, in many practical combustion problems the second domain asymptotically shrinks into the boundary between first and third domains (see Figs. 1 and 2b) and, consequently, can be ignored or neglected without essential loss of accuracy. This might be 

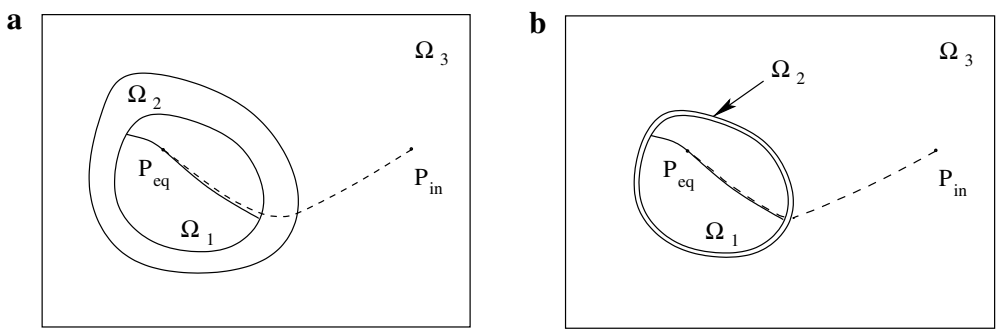

Fig. 2. Sketch of the phase space with asymptotical domains.

considered as an additional assumption, but for real combustible mixtures it is fulfilled automatically due to strongly nonlinear dependence of the chemical term on system quantities. Moreover, the problem with accuracy can be solved by increasing the dimension of the considered manifold. In fact, it is a rare situation that there are many slow chemical modes that have to be taken into account for interaction with physical modes in the whole domain of interest in the state space, at least for typical combustion systems.

In the following, let us concentrate on the most complicated and typical case when the initial and equilibrium points belong to different domains. Now, the basic idea is to exploit the behaviour in the different domains and to construct a matching procedure between the different domains. Specifically, in the first domain $\Omega_{1}$ the ILDM can be applied to obtain the reduced dynamics and then extended to the third $-\Omega_{3}$. To understand what might be used as a suitable extension of the ILDM, consider the system dynamics in the third domain with an absence of the source term and with the additional assumption of equal diffusivities. In this case the nature of the diffusion term gives rise to the fact that each linear manifold in the composition space constitutes an invariant manifold. In the case of non-equal diffusivities, the situation is more complex, but a similar (non linear) invariant manifold can be obtained by an analysis of the eigenspaces of the diffusion matrix [20]. In other words, the system in Eq. (1) becomes linear, and therefore, any linear combination would be invariant under the degenerated system without the source term. Additionally, the fact that in the stationary limit the system solution is close to the so called mixing line allows us to use locally linear manifolds starting from the boundary domain till the initial/unburned point. The term linear only means that the extended manifold locally belongs to a linear hyper-plane joining the boundary of the ILDM with the initial point.

\subsection{Definition of the boundary manifold}

The next problem that must be solved during the identification of the ILDM is the identification of the boundary separating the two domains. It is overcome by using either the gap condition with some user specified small parameter in Eq. (2) reflecting the difference in time scales of the neglected sub-processes or by defining the turning manifold of the approximated system according to the standard SPS approach [5-8] and [17-19]. Thus, the fact that the point $\psi$ belongs to the boundary or not depends on the following conditions:

$$
\text { (ii) } \psi: \operatorname{Re}\left[\lambda_{i}\left(\tilde{Z}_{\mathrm{f}}\left(\psi_{0}\right) F_{\psi}(\psi) Z_{\mathrm{f}}\left(\psi_{0}\right)\right)\right]<0 \text {. }
$$

In other words, the tabulation procedure for the ILDM continuously checks these inequalities and thus identifies the boundary manifold. The first condition normally reflects the situation when an additional chemical mode becomes slow and therefore the dimension of the slow subsystem has to be increased (see a gap condition introduced in Eq. (2)). The second condition in Eq. (7) means that the system trajectory leaves the low $n_{\mathrm{s}}$-dimensional manifold by relatively fast motion and consequently the dimension has to be increased as well. It is obvious that at this point the questions of definition of the boundary manifold, measuring time scales of the sub-processes as well as determination of the minimal dimension of the slow manifold in the fast chemistry domain are crucial points of the method and must be investigated before generating the matched manifold. Complete answers on these questions can not be given in the general case; they strictly depend on local properties of the considered model and on the accepted level of reduced model accuracy, but they can be obtained during the generation of the ILDM. Therefore future work will focus on an identification of the physical time scales during the integration of the reacting flow equations and on an adaptation of the dimension of the manifold. This can be done by a "calculation of the ILDM on demand" during the flame simulation.

\subsection{Identification and matching of the slow chem- istry manifolds}

Now, assuming that the boundary manifold has been already found, let us describe the 
proposed algorithm of extension of the manifold from the first to the third domain in more details. As it was described earlier a manifold consisting of local hyper-planes and joining the $\left(n_{\mathrm{s}}-1\right)$ dimensional boundary manifold with the boundary point is used in the slow chemistry domain. Accordingly, after reaching the boundary manifold, the ILDM equations (see Eq. (4) or (5)) are replaced by a new set of equations. The algorithm used for the generation of the ILDM is based on a multi-dimensional continuation process [12]. In the slow chemistry domain a linear hyper-plane is constructed locally during the build-up of the manifold cells. In order to allow a simple modification of the existing algorithm, we replace the manifold equations:

$P \psi=0$,

$\tilde{Z}_{\mathrm{f}}(\psi) F(\psi)=0$,

by

$P \psi=0$,

$P^{\perp}\left(\mathrm{I}-\mathrm{VV}^{\perp}\right)\left(\psi-\psi_{\text {in }}\right)=0$,

where $P, P^{\perp}$ denote the parameterization matrix and its orthogonal complement, $I$ is the identity matrix, $\psi_{\text {in }}$-the initial point and $V=$ $\left(\psi-\psi_{\text {in }}\right) e+T$, here $e$ is the unit vector, $e=(1, \ldots, 1)^{T}$, and $T$ is the local tangent space of the manifold boundary (see [11] for details of the manifold construction). It can be shown by simple algebra that all points fulfilling Eq. (9) lie on the linear hyper-plane spanned by the cell boundary and initial point.

\section{Implementation}

In reality one has to make a choice between the cost of integrating the system dynamics on the relatively high dimensional manifold and accuracy needed for simulations. If it is important to approximate the detailed chemistry without a high accuracy, a low dimensional manifold of dimension one or two might be considered in the fast chemistry domain.

If a more accurate description of detailed chemistry throughout the flame front is needed then a manifold of relatively high dimension has to be used. Moreover, the use of a high dimension might sufficiently improve the situation with the boundary domain when the boundary domain separating the dynamics is not negligible. Of course the calculation of high dimensional ILDMs may be computationally expensive, but in practical for higher dimensional manifolds approximations for the invariant manifold can be used. Such approximations which has been used here, is described above.

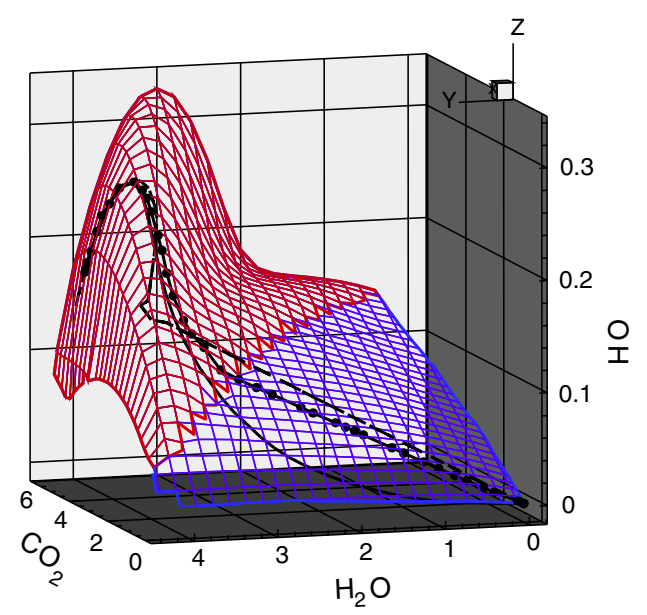

Fig. 3. Projection of the state space onto $\mathrm{CO}_{2}-\mathrm{H}_{2} \mathrm{O}-\mathrm{OH}$ space. Solid lines are stationary solutions for detailed kinetics, reduced by 1D and 2D modified ILDMs. Mesh shows the extended 2D ILDM.

To summarize let us repeat shortly the main steps of the suggested algorithm:

(1) Generation of the ILDM according to Eqs. (8), (5) or (6) depending on the accepted level of accuracy);

(2) Definition of the boundary manifold by using the boundary condition: Eq. (7);

(3) Calculation of the approximation in the slow chemistry domain from the boundary till the initial point Eq. (9);

(4) Tabulation of the extended ILDM together with information on the state space and the projection operator for the transport term;

(5) Use of the tables in flame calculations.

Figure 3 visually represents the major steps in projection onto 3D space. It can be seen that on the active chemistry part the modified ILDM (the first order approximation was used according to the specification given in Section 2.1) approaches the stationary solution quite good, then it reaches the boundary and after that almost linear extension is built up.

\section{Results: $\mathrm{CO} / \mathrm{H}_{2} / \mathrm{H}_{2} \mathrm{O} / \mathrm{CO}_{2} / \mathrm{N}_{2} / \mathrm{O}_{2}-$ premixed laminar flames}

To illustrate the approach in details and to show special features of considered combustion problems 1D stationary free flat flame structures have been calculated using detailed chemical kinetic model. In order to simplify the presentation, the main physical assumptions are as the following: constant pressure, equal diffusivity, Lewis 

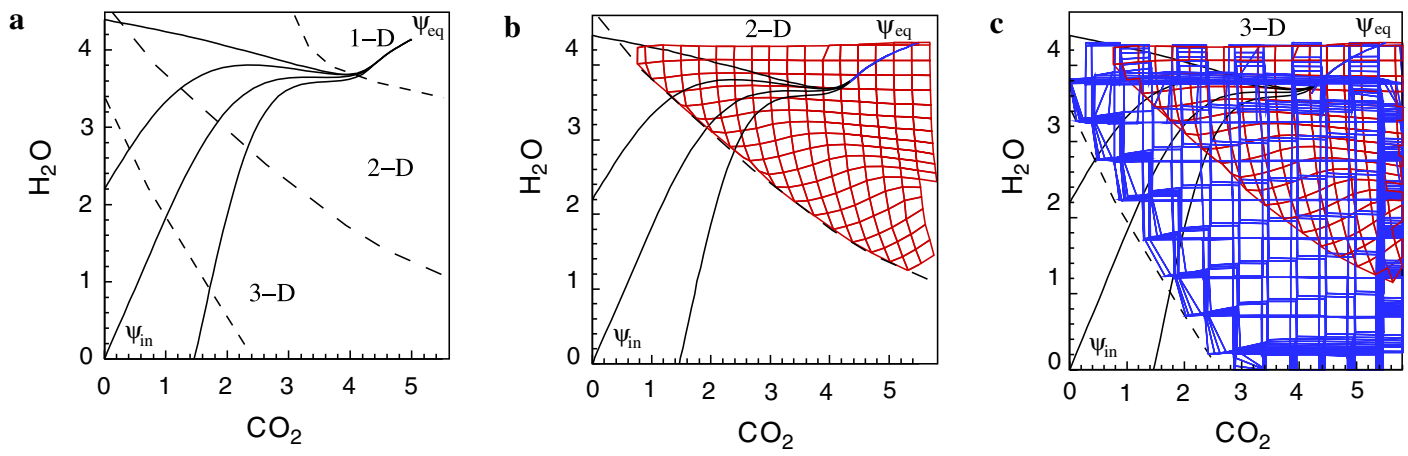

Fig. 4. Projections of the state space onto the $\mathrm{CO}_{2}-\mathrm{H}_{2} \mathrm{O}$ planes. Solid lines are stationary solutions, dashed lines are projections of the boundary manifolds, and mesh in (b) is the projection of the 2D ILDM, mesh in (c) is the projection of the 3D ILDM.

number equals to unity. (The general case of more complicated transport models had been the subject of previous earlier publications [11].)

\subsection{State space analysis}

The example is a laminar flame of carbon monoxide/hydrogen/air mixture $\left(\mathrm{CO} / \mathrm{H}_{2} / \mathrm{H}_{2} \mathrm{O} / \mathrm{CO}_{2} /\right.$ $\left.\mathrm{N}_{2} / \mathrm{O}_{2}\right)$. In this case the state space is 15 -dimensional, and the dimension of the reaction space is 9. Figure 4 shows projection of the 1D-, 2Dand 3D ILDM manifolds together with the stationary solutions for different boundary conditions. The state space is projected onto the $\mathrm{CO}_{2-}$ $\mathrm{H}_{2} \mathrm{O}$ plane. These variables are often used to parameterize the ILDM manifold because they are major reaction products. As one can see the projected part of the phase plane might be roughly subdivided according to the curves plotted in the figure. Namely, near the initial/unburnt and equilibrium/burnt points (Figs. 4a and b) all the system trajectories follow the straight mixing lines starting at the initial point and 1D ILDM near equilibrium, then being far away from those points and close to the boundary domain they start to deviate from the both curves: the system trajectory leaves the 1D ILDM manifold and furthermore it is no longer a straight line far away from the initial point. Because the chosen variables for projecting are relatively slow their modes are strongly coupled with convection/diffusion processes in the boundary domain which becomes to be no longer negligible.

The boundary domain does not shrink to the boundary manifold, at least on this projection see in contrast Fig. 1, therefore the use of a 2D manifold improves the accuracy of the reduced model (Fig. 4b). Figure 4c finally shows that the 3D manifold covers the whole domain, where a deviation from the linear mixing process is observed. In addition, Fig. 5 provides further qualitative information about the $3 \mathrm{D}$ structure

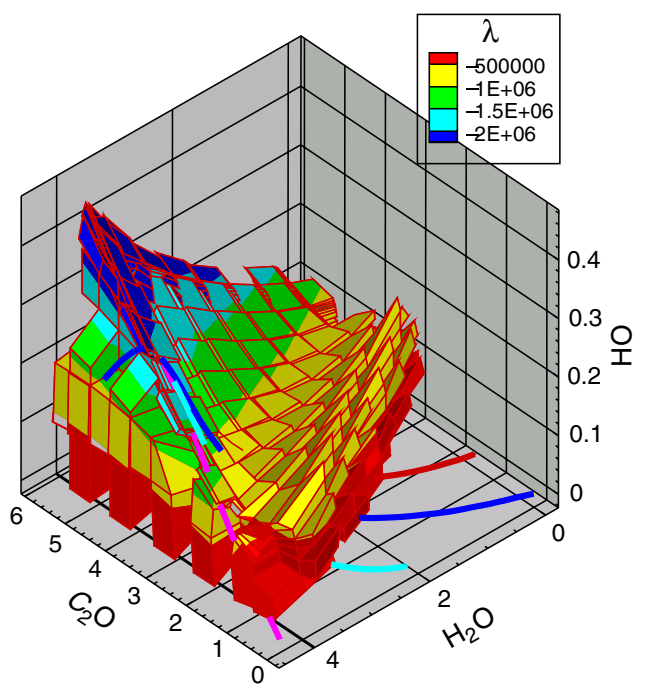

Fig. 5. Projections of the state space onto $3 \mathrm{D}\left(\mathrm{CO}_{2-}\right.$ $\left.\mathrm{H}_{2} \mathrm{O}-\mathrm{OH}\right)$ space. Solid lines are stationary solutions starting at different initial points and having the same element composition and enthalpy. Bricks show the 3D ILDM.

of the modified ILDM. The contour shows by the real values of smallest eigenvalue of the fast subspace. One can see that the typical time scales of the fast chemical sub-processes are faster than typical time scales of physical processes.

\subsection{Comparison of detailed and reduced models}

Let us now present the performance of the extended 1D and 2D ILDMs. We continue with the same example, but consider only one mixture composition and extend the 1D and 2D dimensional manifold according to the suggested method. The results of the extension on the whole domain 
$\mathbf{a}$
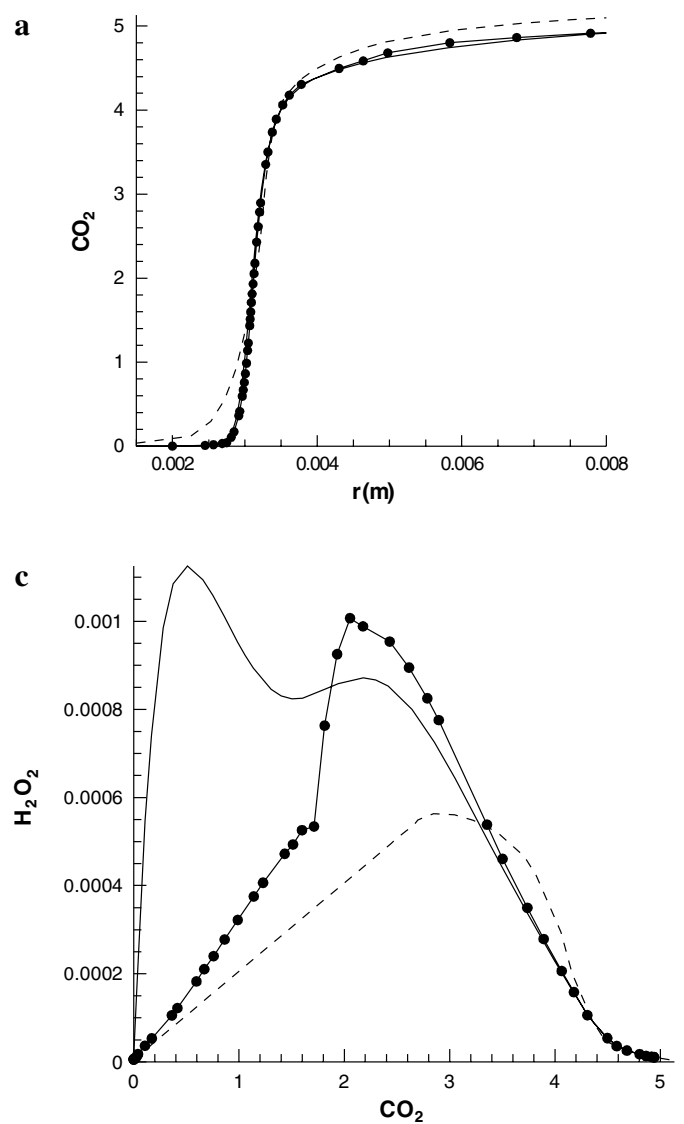

b

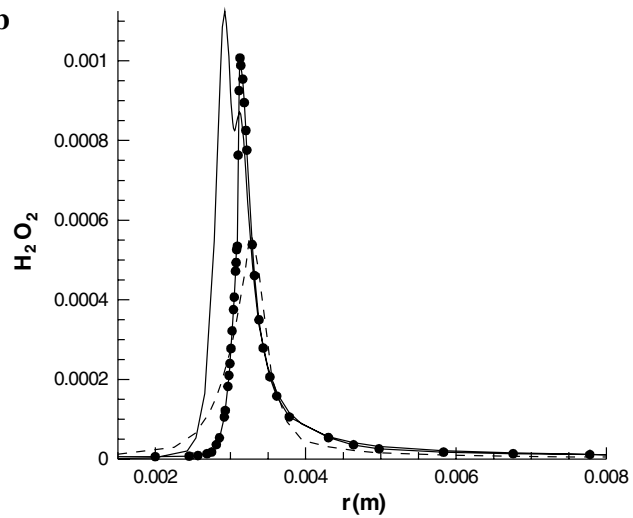

d

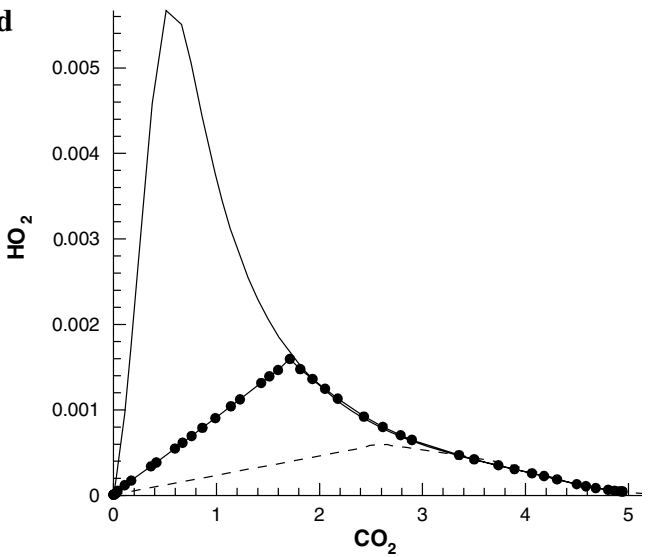

Fig. 6. Stationary profiles of major species $\mathrm{CO}_{2}$ (a) and minor $\mathrm{H}_{2} \mathrm{O}_{2}$ (b) and projections of the stationary solutions onto the $\mathrm{CO}_{2}-\mathrm{H}_{2} \mathrm{O}_{2}$ plane (c) and onto the $\mathrm{CO}_{2}-\mathrm{HO}_{2}$ plane (d). Solid line, detailed calculation; dashed line, the stationary solution based on the 1D extended ILDM, solid line with circles is the stationary solution using the 2D modified ILDM.

are shown in Fig. 3. In Fig. 6 the results of the use of reduced models are presented by typical profiles of major $\left(\mathrm{CO}_{2}\right.$, Fig. 6a) and minor $\left(\mathrm{H}_{2} \mathrm{O}_{2}\right.$, Fig. 6b) species and by projections of the stationary solutions onto the planes of the $\mathrm{CO}_{2}-\mathrm{H}_{2} \mathrm{O}_{2}$ (c) and the $\mathrm{CO}_{2}-\mathrm{HO}_{2}$ (d) planes, respectively. The solid lines denote the detailed calculations, dashed lines show the stationary solution of reduced system based on the 1D extended ILDM, and the solid lines with filled circles represent the stationary solution when the 2D ILDM is used. It is not hard to see that although the extended 2D ILDM increases considerably the accuracy of the reduced model from both detailed chemical and hydrodynamics aspects there is a problem to quantitatively describe some minor species and therefore the dimension has to be increased at least by one (see Figs. 4c and 5) if an accurate description of some minor species in the slow chemistry domain is needed. Finally, it has to be mentioned that in the present work we chose simple test cases of ILDMs of dimension 1 and 2. In previous work (see e.g., [14]) we showed that minor species can be predicted very well by 3D manifolds.

\section{Conclusions}

A method for constructing of the approximation of the manifold of slow motions in the whole domain of interest in the state space of complex combustion problem has been presented and discussed. It is based on natural assumptions widely used in combustion theory. Namely, it is assumed that there exists a sharp subdivision (splitting) of the state space into sub-domains with predomination of chemical kinetics or convection/diffusion terms. Accordingly, in the domain of fast chemistry the ILDM method or its suggested modification is used to approximate invariant manifold of slow motions whereas in the "no chemistry" or low-temperature domain the locally linear extension of the ILDM and matching procedures have been proposed. The boundary is identified by 
combined analysis of the local Jacobian and relevant constant decomposition structure. It is suggested to increase the ILDM dimension to improve the performance of the ILDM near the boundary manifold and an approximation of the IDLM has been suggested in order to speed-up the generation procedure for the ILDM table. The 1D premixed adiabatic syngas/air free flames with detailed and reduced chemical mechanism were used to verify the proposed approach. Calculations show a good asymptotical agreement of the system dynamics on the constructed manifold with full system solutions.

\section{Acknowledgments}

Financial support by the Minerva Fellowship Program of the Max Planck Society is gratefully acknowledged. This research was partly supported by the Grant G-695-15.10/2001 from the G.I.F., the German-Israeli Foundation for Scientific Research and Development.

\section{References}

[1] S.H. Lam, D.M. Goussis, Int. J. Chem. Kinet. 26 (1994) 461-486.

[2] M. Hadjinicolaou, D.M. Goussis, SIAM J, Sci. Comput. 20 (3) (1999) 781-910.

[3] M. Valorani, D.A. Goussis, F. Creta, H.N. Najm, J. Comp. Phys. 209 (2) (2005) 754-786.

[4] A.S. Tomlin, T. Turanyi, M.J. Pilling, in: M.J. Pilling (Ed.), Mathematical tools for the construction investigation and reduction of combustion mechanisms, Comprehensive Chemical Kinetics 35: Lowtemperature Combustion and Autoignition, Elsevier, 1997.

[5] N.N. Bogolyubov, Yu.A. Mitropolsky, Asymptotic Methods in the Theory of Nonlinear Oscillations, N. Y. Gordon and Breach, 1961.

[6] N. Fenichel, J. Different. Equat. 31 (1979) 53-98.

[7] B.B. Strygin, V.A. Sobolev, Decomposition of Motions by the Integral Manifolds Method, Moscow, Nauka (in Russian), 1988.

[8] V. Gol'dshtein, V. Sobolev, Singularity Theory and Some Problems of Functional Analysis, AMS Translations, Series 2, 153 (1992) 73-92.

[9] M.R. Roussel, S.J. Fraser, Chaos 11 (1) (2001) 196206.

[10] U. Maas, S.B. Pope, Combust. Flame 117 (1992) 99116.

[11] U. Maas, S.B. Pope, Proc. Combust. Inst. 25 (1994) $1349-1356$.

[12] U. Maas, Comput. Visual. Sci. 1 (1998) 69-82.

[13] J. Warnatz, U. Maas, R.W. Dibble, Combustion, Springer Verlag, 2000.

[14] J. Nafe, U. Maas, Combust. Flame 135 (2003) 17-26.

[15] C. Rhodes, M. Morari, S. Wiggins, Chaos 9 (1) (1999) 108-123.

[16] H. Kaper, T. Kaper, Argonne National Lab, preprint ANL/MCS-P912-1001, 2001.

[17] I. Goldfarb, V. Goldstein, U. Maas, IMA J. Appl. Math. 69 (2004) 353-374.

[18] V. Bykov, I. Goldfarb, V. Gol'dshtein, U. Maas, IMA J. Appl. Math. 82 (2005) 1-24.

[19] V. Bykov, U. Maas, in: Proceedings of the 20th International Colloquium on the Dynamics of Explosions and Reactive Systems, 2005, p. 244.

[20] V. Bykov, U. Maas, Combust. Theory Modell. (2006), Submitted for publication.

[21] J.A. van Oijen, L.P.H . de Goey, Combust. Theory Modell. 6 (2002) 463-478. 